\title{
Error Rate Performance in High Data Rate UWB Channels for Wireless Personal Area Networks
}

\author{
Dr Jyoteesh Malhotra \\ Department of Electronics and Communication Engineering \\ Guru Nanak Dev University Regional Campus, \\ Jalandhar, India
}

\begin{abstract}
The error rate link performance has been evaluated and compared for efficient Rake receiver structures in high data rate ultra-wide band (UWB) realistic channels. Exponential-Lognormal model for high data rate UWB indoor channels has been used for generating Power Delay Profiles (PDP). This model is based on extensive measurements in diversified Residential \& Commercial environments. The PDPs for CM1 (0-4m), CM2 (0-4m) and CM3 (4$10 \mathrm{~m})$ UWB channel categories have been generated. The probability density functions (PDF) have been obtained through discrete realization in all channel categories. With the aid of PDFs, quasianalytical error rate performance evaluation of sub-optimum Rake receivers has been done. Through simulative investigations of the outage probability and Average Bit Error Rate, it has been found that the low complexity (optimum number of fingers) partial Rake receiver is almost as good as the selective Rake receiver in high data rate UWB channels.
\end{abstract}

\section{Keywords}

Ultra-Wide Band, Wireless Personal Area Network, Outage Probability, Average Bit Error Rate, Power delay profile, BiOrthogonal Keying.

\section{INTRODUCTION}

ULTRA wideband (UWB) wireless transmission is ideally suited for short range, high speed Wireless Personal Area Networks (WPANs). Commercial interest in UWB techniques increased significantly after the Federal Communications Commission allowed unlicensed UWB communications. In 2002, the Task Group 3a was established within the IEEE 802.15 to define a standard for high data rate communication systems. The potential strength of the UWB radio technique lies in its use of extremely wide transmission bandwidth and limited power spectral density that results in host of desirable capabilities including high multipath resolvability, accurate position location and ranging, lack of significant fading, high multiple access capability, and possible easier material penetration[1-3]. In order to build systems that realize UWB potential, it is first required to understand UWB propagation and the channel properties arising from its propagation. The choice of Rake receiver structure and the relative performance of different modulations also depend on the propagation channel. There has been a great deal of activity to characterize the UWB propagation channel [4-7]. Three delay profile models widely referenced in the literature are Stochastic tapped delay line model [4], multi-cluster model [5-6] and Exponential-Lognormal model [7]. Exponential-Lognormal model based on extensive measurements has been selected here to evaluate the WPAN system due to its merits. The Exponential-Lognormal model gives best fit to measured data statistics than the Cluster model as reported in [8], because its numeric parameters are derived from the extensive measurement database. As such this model will provide realistic results for the system performance analysis. Moreover, the propagation channel also influences such design aspects as construction of the matched filter, modulation format, and choice of the Rake receiver structure [9]. Performance analysis of WPAN systems was done with Stochastic Tap Delay Line model to understand low data rate UWB propagation channel properties in [10]. Moreover, the performance of high data rate UWB radio channel has been reported for CM1 and CM2 [11]. In this paper, the effect of the UWB channel characteristics on the Rake receiver performance has been quantified through performance measures such as Probability Density function (PDF) of output SNR, outage probability and Average Bit Error Rate (ABER). The different number of Rake fingers have been tried to find the optimum energy capturing performance of receivers in CM1, CM2, and CM3 channels.

This paper is organized as follows. In the next section, brief description of UWB channel model along with related parameters has been given. Subsequently, the Simulation methodology to generate PDPs in UWB channel scenarios has been described. In Section 4, sub-optimum reduced complexity Partial Rake (PRake), Selective Rake (SRake) receiver architectures have described along with ideal All Rake (ARake) employing Maximal Ratio Combining (MRC). Generated profiles of realistic Exponential-Lognormal model for CM1, CM2 and CM3 Channel scenarios have been presented along with their respective PDFs. Error rate investigations have also been presented in terms of outage probability and ABER, followed by Section 6, wherein we conclude the paper.

\section{UWB CHANNEL MODEL}

The impulse response of the channel $h t$ can be written as

$$
h t=\sum_{n=0}^{N t-1} a_{n} t \delta t-\tau_{n} t
$$

where $a_{n} t$ is the amplitude fading statistics, $\tau_{n} t$ is the arrival time delay, $N t$ is the number of paths at time $t$ for the $n$th path and $\delta t$ is the dirac delta function. The parameters characterizing the impulse response (1) of the UWB channel are time-varying due to the movement of either transmitter or receiver. Because this time rate of change is slow compared to the pulse rate the channel can be assumed to be stationary over an observation time. With this assumption (1) can be rewritten as

$h t=\sum_{n=0}^{N-1} a_{n} \delta t-\tau_{n}$

A convenient and simple model for characterization of UWB channels is the discrete time, multipath, impulse response model [11]. 
In this model the delay axis is divided into small time intervals called bins. Each bin is assumed to contain either one multipath component or no component. The possibility of more than one path in a bin is excluded. A reasonable bin width is the largest time interval over which two separate paths cannot be resolved as distinct paths. Using (2) the discrete time channel model can be represented as

$h t=\sum_{n=0}^{N_{\max }-1} a_{n} \delta t-n \Delta \tau$

where $N_{\max }$ is the maximum number of bins or resolvable multipath components considered within a observation interval are important because they determines the design of a rake receiver, $\Delta \tau$ is the time duration of the bin equal to the UWB pulse period for which it is non-zero. Equation (3) is referred to as multipath intensity profile (MIP). Using (3), the received signal power can be obtained as

$$
P t=E\left[|h t|^{2}\right]=\sum_{n=0}^{N_{\max }-1} E\left[a_{n}^{2}\right] \delta t-n \Delta \tau
$$

which represents the PDP of impulse response which shows the time of arrival ' $n$ ' of different contributions versus received power and $E\left[a_{n}^{2}\right]$ is the average received power by the $n$th bin. Usually the later bins of PDP captures less energy due to higher path attenuations and accordingly the PDP is a decreasing function of excess delay. The total multipath gain $G$ that measures the total amount of energy collected over the $N_{\max }$ bins with unitary energy transmitted as

$$
G=\sum_{n=0}^{N_{\max }-1}\left|a_{n}\right|^{2}
$$

The MIP will be written in terms of multipath gain as

$$
h t=\sqrt{G} \sum_{n=0}^{N_{\max }-1} \alpha_{n} \delta t-n \Delta \tau
$$

where $\alpha_{n}$ is energy normalized channel gain parameter such that $\sum_{n=0}^{N_{\max }-1}\left|\alpha_{n}\right|^{2}=1$. The value of $G \leq 1$ is due to attenuation during propagation and it decreases with distance according to $G=G_{0} / d^{\gamma}$, where $G_{0}$ is the reference gain at distance $d=1 \mathrm{~m}$ that can be evaluated as $G_{0}=10^{-A_{0} / 10}$ in terms of parameter $A_{0}(\mathrm{~dB})$ and $\gamma$ is the decay exponent. For the UWB wireless channels single-number parameter of an impulse response profile is the RMS delay spread given as

$\tau_{r m s}=\sqrt{\overline{\tau^{2}}-\bar{\tau}^{2}}$

where $\bar{\tau}$ mean excess delay is the first moment and $\overline{\tau^{2}}$ is the second moment of PDP, respectively, given as

$$
\overline{\tau^{2}}=\sum_{n=0}^{N_{\max }-1} \tau_{n}^{2}\left|a_{n}\right|^{2} / G \text { and } \bar{\tau}=\sum_{n=0}^{N_{\max }-1} \tau_{n}\left|a_{n}\right|^{2} / G
$$

RMS delay spread is a good measure of signal spread due to multipath channel. As can be seen from (7) strong echoes with long delays contribute significantly to $\tau_{r m s}$ and the performance of UWB systems is very sensitive to the value of $\tau_{r m s}$.

\section{SIMULATION METHODOLOGY}

It is necessary to design the receiver using measurement based realistic channel model since performance analysis of the receiver is based on statistics of the channel. The simulation model used here to statistically characterize the PDP of UWB channel that is based on an exponential profile multiplied by a noise-like variation with lognormal statistics has been briefly describe here. The PDP in general form is given by

$$
P t=\sum_{n} P_{n} \delta t-\tau_{n}
$$

where $\sum_{n} P_{n}=1$. Thus, the PDP is described by the power-delay set $P_{n}, \tau_{n}$. In a given bandwidth, $B$ sampling theory explains that the PDP is characterized via a set of samples spaced by $1 / B$ (i.e. $\left.\tau_{n}=n / B n \in 1,2 \ldots\right)$, and the result is suitable for any bandwidth of $B$ or smaller. There are two versions of the multipath delay profile generally used i.e. one corresponds to PDP at a fixed point receiver and other is locally (spatially) averaged PDP [4]. The latter type also called as small scale averaged PDP has been used in this work. In the Exponential- Lognormal model given in [7] the PDP for NLOS paths varies with delay as a decaying exponential times a noise-like variation that behaves as a correlated lognormal random process, i.e.

$$
P_{n}^{n l o s}=k \cdot e^{\left(-\frac{\tilde{\alpha} \cdot \tau_{n}}{\bar{\tau}_{m s}}\right)} \cdot s \quad \tau_{n} \quad n \geq 0
$$

The PDP when strong LOS exists between transmitter and receiver paths, there is an additional term at the minimum delay followed by an exponential-lognormal term i.e.

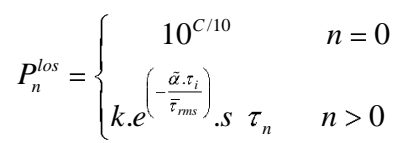

where $\tilde{\alpha}$ is a decay constant and $C$ is the direct (LOS) ray amplitude both varies with $\mathrm{Tx}-\mathrm{Rx}$ distance $d ; s \tau_{n}$ is a noise-like variation with delay behaves like a correlated lognormal process; $\bar{\tau}_{r m s}$ is a global average of the RMS delay spread; and $k$ is a normalizing factor such that $\sum_{n} P_{n}=1$. The dB value of $\tilde{\alpha}$ parameter will be

$\alpha=\alpha_{0}-\gamma \cdot \log _{10} d+\varepsilon_{\alpha}$

where $\alpha_{0}$ is a numeric constant; $\varepsilon$ is a zero-mean Gaussian random variable with standard deviation $\sigma_{\alpha}$ and $\gamma=\bar{\gamma}-2, \bar{\gamma}$ is a gamma distributed RV given in [7] using fitting parameters $u$ and $v$. $\varepsilon_{\alpha}$ is the zero mean normal distributed from one path to another with standard deviation $\sigma_{\alpha}$. The distance dependent LOS amplitude in $\mathrm{dB}$ 
$C=A_{0}-10 \cdot \gamma_{A} \cdot \log _{10} d+\varepsilon_{C}$

where $A_{0}$ and $\gamma_{A}$ are constants, $\varepsilon_{C}$ is a zero-mean Gaussian random variable with standard deviation $\sigma_{C}$. The $\mathrm{dB}$ value of lognormal variation parameter $s \tau_{n}$ is

$S_{n} \tau_{n}=\sigma_{s}\left[\sigma_{0} . e^{\left(\frac{-n \beta}{B \cdot \bar{\tau}_{m n s}}\right)}\right] . x_{n} n \in 0,1,2, \ldots$

where $\sigma_{s}, \beta_{n}$ and $\sigma_{0}$ are constants, $x_{n}$ is a zero-mean correlated Gaussian sequence having the following autocorrelation function

$\rho_{x} \Delta n=\left\{\begin{array}{cc}1 & \Delta n=0 \\ a . e^{\left(\frac{-|\Delta n| \cdot b}{W \cdot \bar{\tau}_{m s}}\right)} & \Delta n \neq 0\end{array}\right.$

where $\rho_{x} \Delta n$ is the correlation coefficient between two values of $x_{n}$, separated by $\Delta n$. The values of $a$ and $b$ constants are given in [7]. The correlated Gaussian sequence $x_{n}$ can be generated using the following linear difference equation [7] as

$x_{n}=Q x_{n-1}+\sqrt{E} w_{n}-\sqrt{E} D w_{n-1}$

where $Q=\exp -b / B \bar{\tau}_{r m s} \quad, \quad E=F+\sqrt{F^{2}-4 H^{2}} / 2 \quad, \quad$ and $D=F-\sqrt{F^{2}-4 H^{2}} / 2 H$ are the linear coefficients. $w_{n}$ is the white Gaussian sequence, $F=1+Q^{2} 1-2 a$ and $H=Q 1-a$.

\section{RECEIVER STRUCTURE}

Given the characteristics of the propagation UWB channel, different multipath components MPCs of the same transmitted pulse do not overlap if the inter-arrival time is greater than the UWB pulse duration and each of the resolved MPCs can be assumed to be independently fading. Moreover, the smaller pulse duration gives higher number of independent contributions ' $N_{R}$ ' at the receiver input, thereby providing a high degree of temporal diversity [1]. Thus, UWB receiver can exploit this diversity using Rake combiner to improve performance of the system. The basic version of the Rake receiver consists of multiple correlators (fingers) where each of the fingers can detect/extract the signal from one of the multipath components provided by the channel. The outputs of the fingers are appropriately weighted and combined to reap the benefits of multipath temporal diversity. Different strategies for exploiting this temporal diversity include Selection Diversity, Equal Gain Combining and MRC. In single user communication without ISI, the MRC achieves the best performance due to largest SNR at the Combiner output. In MRC Rake receiver, the SNR is maximized by applying to each multipath contribution a weighting factor that is proportional to the channel gain coefficient of the corresponding received signal. Each correlator first aligns the MPCs in time that are assumed to differ by one bin duration and then use the common correlators mask before applying to the integrator. The ARake structure used here to indicate the receiver with unlimited resources (fingers or correlators) and instant adaptability, so that it can, in principle, combine all of the resolved MPCs. To achieve this, it requires $N_{R}=T_{d} / \Delta \tau$ fingers, where $\Delta \tau$ is the bin duration and $T_{d}$ is the time duration of impulse response. Since the number of resolvable multipath components increases with the spreading bandwidth, the number of correlators required for the ARake receiver may become quite large for UWB channels, typically more than 100 [14]. Realistically, the number of multipath components that can be utilized in a typical Rake receiver is limited by power consumption, design complexity and channel estimation that triggered the need of reduced complexity receivers for the dense multipath UWB channels. Quasi-analytical investigations in [2] showed that a Rake receiver with about 50 fingers captures $60 \%$ of the total energy. Thus, alternative reduced complexity Rake receivers that process only a subset of the available $N_{R}$ resolved multipath components are to be realized. Two sub-optimum reduced complexity Rake structures i.e., SRake and PRake have been proposed here for performance evaluation. The upper limit of achievable performance obtained with ARake receiver will act as benchmark for relative performance comparison. The realistic Exponential-lognormal UWB channel model, as described in the previous Section has been used here for error rate performance. The SRake selects the $N_{b}$ best paths (a subset of the $N_{R}$ available resolved multipath components) and PRake selects the first $N_{P}$ paths (which are not necessarily the best) then combines the selected subset using MRC. The combiner produces a decision variable at its output which is then processed by a detector. Thus, the detector performance can be considered to be based on this equivalent channel created by the cascade of the radio channel and MRC-Rake structures.

\section{RESULTS AND DISCUSSION}

From the description of the simulation model given in the previous section, the realistic exponential-lognormal model need to be specified with 10 numeric parameters for NLOS condition and 13 numeric parameters for LOS conditions for the indoor channel. These model numeric parameters are derived in [7] for LOS and NLOS channel scenarios, based on extensive measurements over $6 \mathrm{GHz}$ Bandwidth centered around $5 \mathrm{GHz}$.

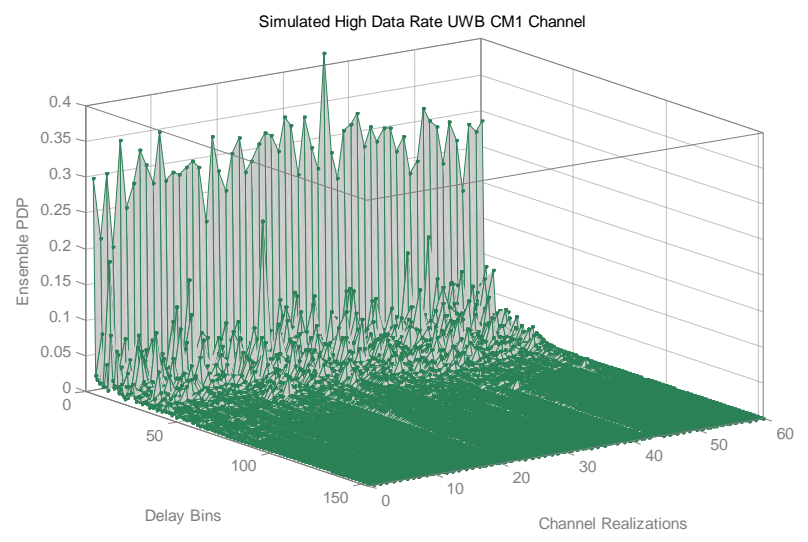

Figure 1. Ensemble PDP in realistic high data rate UWB CM1 channel 
TABLE I. NUMERICAL VALUES OF PARAMETERS USED IN EXPONENTIAL-LOGNORMAL MODEL

\begin{tabular}{|c|c|c|c|c|c|c|c|c|c|c|c|c|c|}
\hline Channel Type & $\alpha_{0}$ & $v$ & $u$ & $\sigma_{\alpha}$ & $A_{0}$ & $\gamma_{A}$ & $\sigma_{C}$ & $a$ & $b$ & $\sigma_{s}$ & $\sigma_{0}$ & $\beta$ & $\bar{\tau}_{r m s}$ \\
\hline CM1 & 3.51 & 3.69 & 0.89 & 1.01 & -4.07 & 1.35 & 0.84 & 0.86 & 0.26 & 4.03 & 2.16 & 0.26 & $3.38 \mathrm{~ns}$ \\
\hline CM2 \& CM3 & 5.29 & 2.72 & 1.58 & 0.84 & -- & -- & -- & 0.73 & 0.15 & 3.68 & 1.77 & 0.19 & $7.31 \mathrm{~ns}$ \\
\hline
\end{tabular}

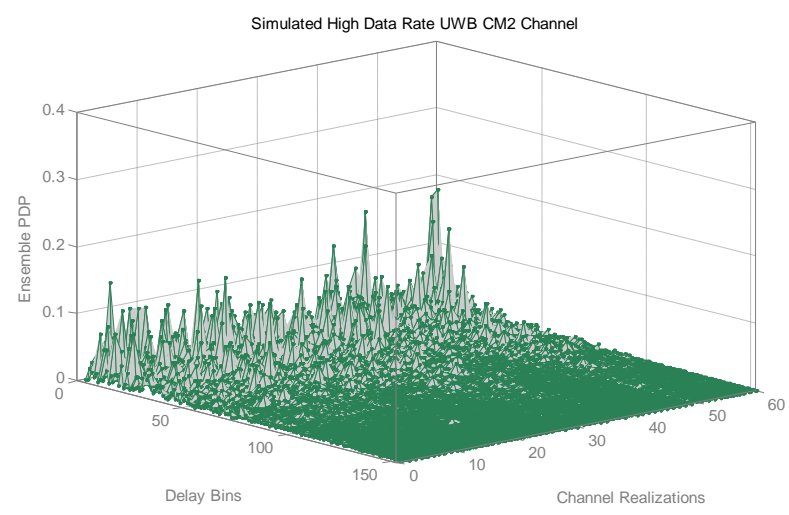

Figure 2. Ensemble PDP in realistic high data rate UWB CM2 channel

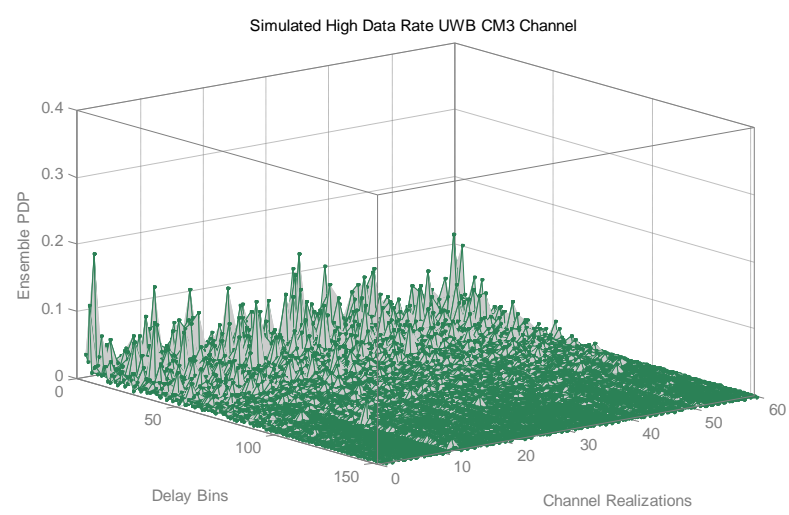

Figure 3. Ensemble PDP in realistic high data rate UWB CM3 channel

Table I enumerates parameters used in generating the PDPs. The channel realizations of PDP for CM1, CM2 and CM3 using Exponential-lognormal model described in previous section are shown in Fig. 1, 2 and 3. The three channel categories generally used for analysis are: Category CM1 consists of LOS paths in residences with 0 $<\mathrm{d}<4 \mathrm{~m}$; CM2 consists of NLOS paths in residences with $0<\mathrm{d}<4 \mathrm{~m}$; and CM3 consists of NLOS paths in residences with $4 \mathrm{~m}<\mathrm{d}<10 \mathrm{~m}$. The CM1, CM2 and CM3 channel conditions are created in Exponential-lognormal model by putting upper ceiling on parameter $\gamma$ as 5.0, 5.6 and 7.6, respectively. The realistic exponential-lognormal model shows exponential decay with no multiple clustering and frequent appearance of LOS component at zero delay in CM1. However, the investigations for the presence of clusters in the measured profile have indicated majority of records with no multiple clusters [7]. Although, large number of numeric parameters are required in Exponential-Lognormal model but they are easy to extract from measured data as reported in [7]. An important virtue of this model is that it accounts for both the frequent presence of a strong LOS component and the fluctuations of the PDP as a function of delay. Also, the generated statistical attributes using this model provides good fit with measured data statistics [8]. Keeping in view, the aforesaid considerations selection of Exponential-Lognormal model has been justified for realistic UWB error rate performance analysis. The first and second order parameters extracted from the generated ensemble of PDP using Exponential-lognormal model in different channel categories are listed in Table II.

TABLE II. EXTRACTED VALUES OF UwB CHANNEL ATTRIBUTES

\begin{tabular}{|c|c|c|c|}
\hline Channel Category & $\begin{array}{c}\text { Mean delay } \\
\text { spread (ns) }\end{array}$ & $\begin{array}{c}\text { RMS delay } \\
\text { spread (ns) }\end{array}$ & $\begin{array}{c}\text { Average no. of } \\
\text { paths within } \\
\text { 10dB of } \\
\text { strongest path } \\
\text { (NP10) }\end{array}$ \\
\hline $\begin{array}{c}\text { CM1 } \\
\text { (LOS 0-4m) }\end{array}$ & 3.5 & 4.01 & 5.8 \\
\hline $\begin{array}{c}\text { CM2 } \\
\text { (NLOS 0-4m) }\end{array}$ & 5.68 & 5.00 & 39.65 \\
\hline $\begin{array}{c}\text { CM3 } \\
\text { (NLOS 4-10m) }\end{array}$ & 6.83 & 5.71 & 48.05 \\
\hline
\end{tabular}

\subsection{Discrete Realizations of PDFs}

The system performance is determined by the probability density function (PDF) of the received SNR. The output SNR in computed by adding the SNR of the selected bins corresponding to each realization of PDP. The discrete realizations of output SNR PDF in different UWB channel conditions are shown in Fig. 4 to 6 . The channel profiles have been generated for normalized channel with unit energy. The average SNR is set to $60 \mathrm{~dB}$ and path loss model of [13] have been used. The transmitter-receiver distance is set to $1 \mathrm{~m}$. Fig. 4 shows the PDF of the output SNR for SRAKE and PRake receivers in the LOS CM1 channel Condition. The two-finger SRake structure performs marginally (about $1 \mathrm{~dB}$ ) better than PRake. This is due to the strong LOS component that frequently appears in the first bin. UWB CM1 channel shows similar difference in diversity gain performance of about $1 \mathrm{~dB}$ with 16 finger structures. Thus, the simple PRake structure performance is very close to SRake in the realistic CM1 channel condition even for lesser number of Rake taps.

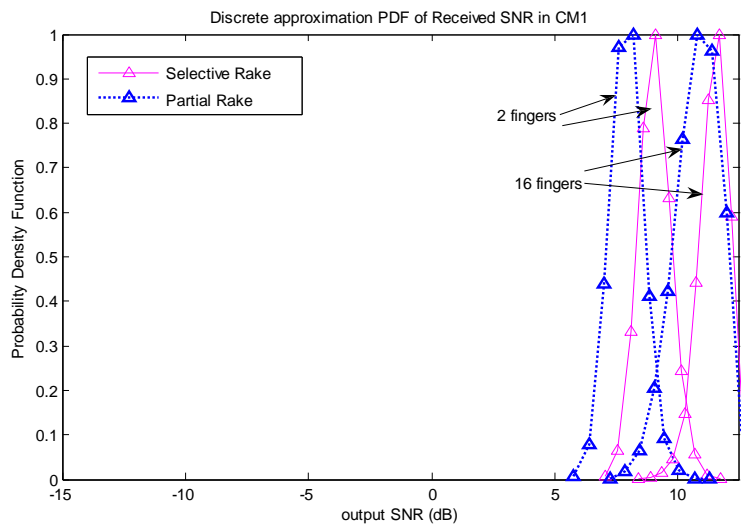

Figure 4. PDF in high data rate UWB CM1 channels

Fig. $5 \& 6$ shows the PDFs of the output SNR for SRake and PRake receivers with different fingers in the both NLOS (CM2 and CM3) 
channel conditions. Due to the absence of strong MPC in the first bin, the average received SNR has been observed to be diminishing in NLOS channel conditions compared with LOS channel scenario. For two finger structures the diversity gain performance gap between SRake and PRake widens in NLOS channel conditions, as the first \& second multipath components may not be the strong components. This degrades the PRake performance more than SRake.

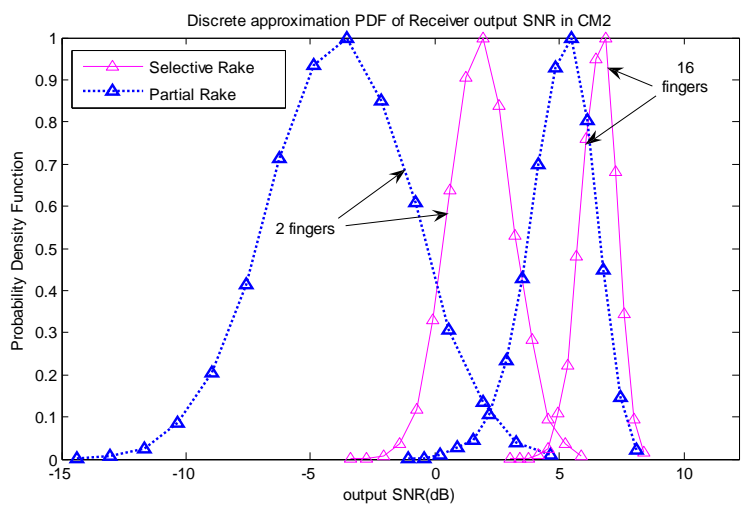

Figure 5. PDF in high data rate UWB CM2 channels

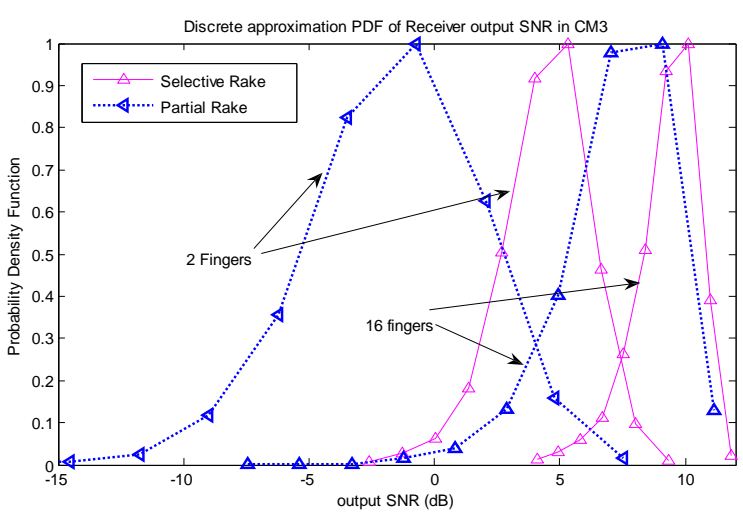

Figure 6. PDF in high data rate UWB CM3 channels

\subsection{Outage Probability}

The OP is a standard performance measure of the quality of the transmission operating over fading channels. The OP can be given as the probability that the receiver performs poorly than a specified threshold BER. For a given modulation technique threshold BER can be mapped into threshold SNR value $\xi$ using conditional error probability formulas. Alternatively, when the received instantaneous SNR $\gamma$ falls below the specified threshold value $\xi$, the error rate drops below the acceptable BER of $P_{e} E$, then OP is the fraction of time during which bit error rate is unacceptable. The OP in terms of threshold SNR value $\xi$ can be calculated as

$$
P_{\text {out }} \xi, \bar{\gamma} \square p_{\gamma} \gamma<\xi=\int_{0}^{\xi} p_{\gamma} \gamma d \gamma
$$

where $p_{\gamma} \gamma$ is the PDF of the output SNR.
Figures 7, 8 and 9 depict the OP performance of SRake and PRake in CM1, CM2 and CM3 channels, respectively. The reference threshold has been computed by considering the path-loss model of [13], while shadowing is normalized. At $10 \%$ OP the threshold SNR gap is about $1 \mathrm{~dB}$ for both 2 and 16 Rake structures in CM1 channel. This is due to the frequent presence of strong MPC in CM1 channel conditions in the first bin and the performance is largely dependent on this component. The slope of performance curve in CM1 shows sharp decrease in OP. The diversity gain indicated by the slope of OP performance curve is less in CM2 channel scenario as shown in Fig. 8. In CM2, the $1 \%$ OP is attained at the threshold SNR of $33 \mathrm{~dB} \&$ $40 \mathrm{~dB}$ in PRake and SRake for two tap structures. This indicates that PRake captures quite weak MPCs in the first two delay bins. Thus, the signal OP performance using PRake structure is quite similar to SRake in CM1 conditions as compared to CM2 \&CM3 channels.

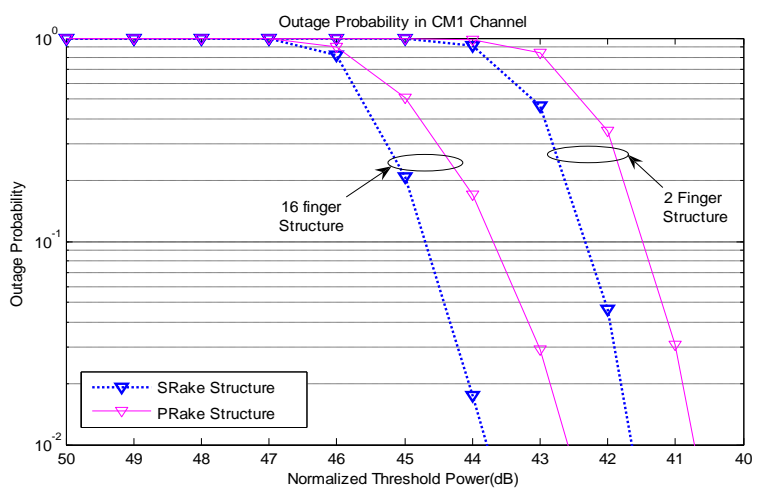

Figure 7. Outage Probability vs threshold SNR in CM1

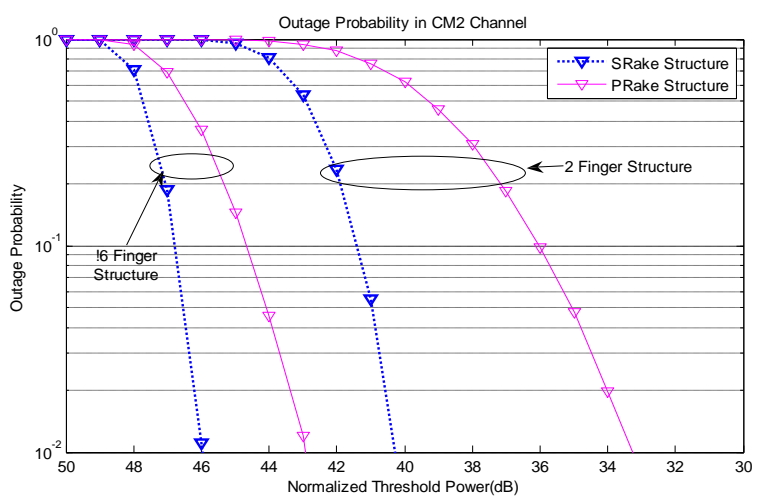

Figure 8. Outage Probability vs threshold SNR in CM2

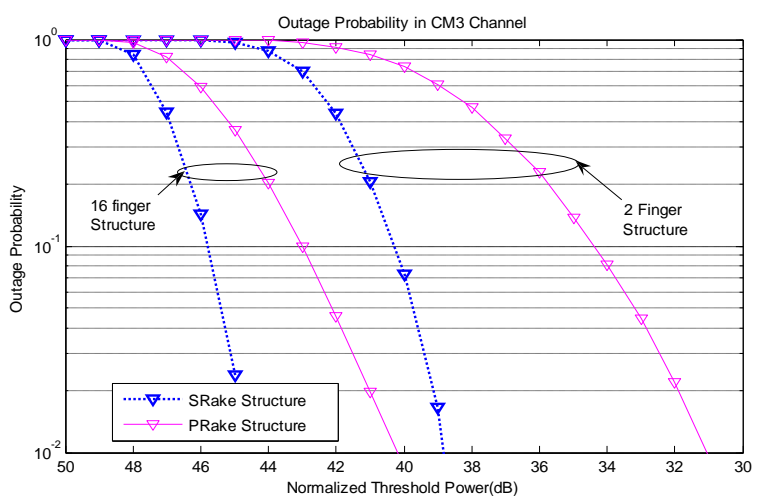


Figure 9. Outage Probability vs threshold SNR in CM3

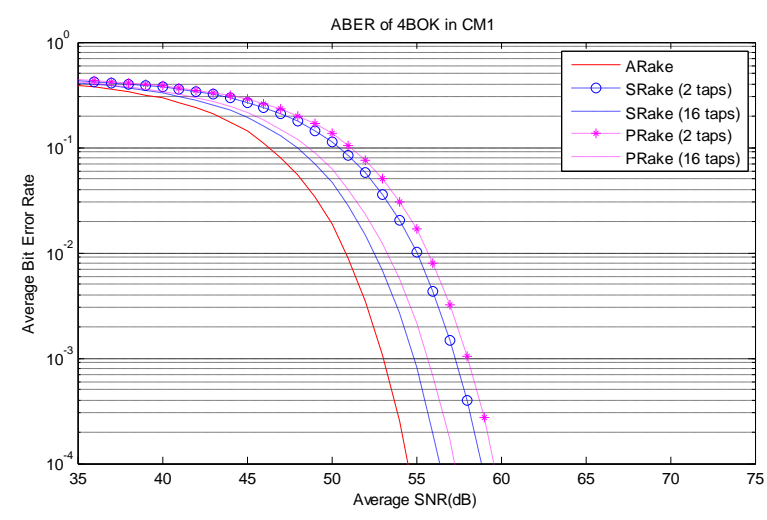

Figure 10. ABER vs SNR in CM1

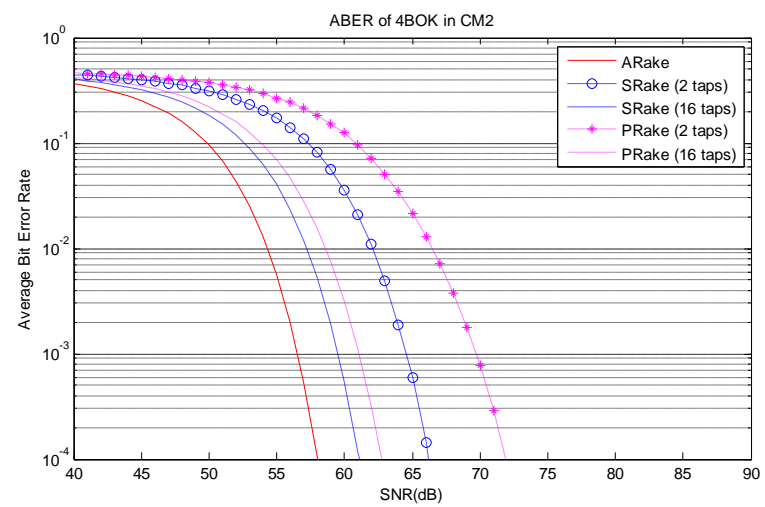

Figure 11. ABER vs SNR in CM1

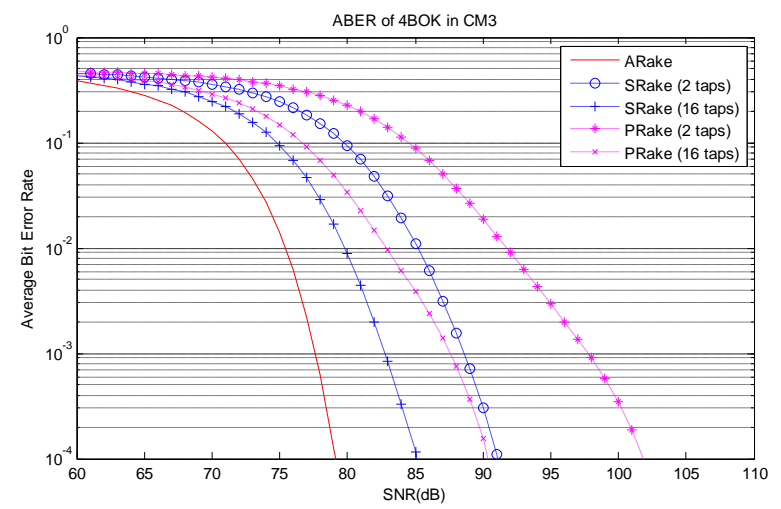

Figure 12. ABER vs SNR in CM1

\subsection{Average Bit Error Rate}

The most common performance measure for the wireless systems subjected to multipath propagation is the ABER. The ABER of SRake and PRake structures is evaluated in the realistic UWB channels and compared with upper performance bound given by ARake. Assuming uncoded, slow fading system with large numbers of bits transmitted within the channel coherence time. In this case, the ABER is obtained by averaging the conditional BER over the PDF of the output SNR at the Rake combiner output [15] as

$$
P_{e} E=\int_{0}^{\infty} P_{e} E / \gamma p_{\gamma} \gamma d \gamma
$$

where $P_{e} E / \gamma$ is the conditional BER and $p_{\gamma} \gamma$ is the PDF of the SNR at the output of Rake combiner. The output SNR depends on the channel conditions and the structure of the Rake receiver. In this analysis, the output from multiple fingers is combined using MRC technique that gives optimum SNR for noise-limited systems with ideal autocorrelation properties of the spreading sequence and channel estimation of the receiver. For the ARake and PRake receivers, the output $\mathrm{SNR}$ is the sum of $N_{R}$ and $N_{P}$ independent and non-identically distributed SNRs of the bins, respectively. But, in the case of the SRake receiver, the best $N_{b}$ SNRs are selected amongst the available multipath components, which mean complete estimation of the channel and SNR at the combiner output is given by the sum of ordered selected SNR random variables. In UWB channel captured SNR in a bin follow a different stochastic distribution that has been obtained in the previous sub-section using discrete realizations of the channel. A quasi-analytical calculation of the bit error probability has been obtained by averaging $P_{e} E / \gamma$ over the channel ensemble (500 realizations of the channel delay profiles for each value of average SNR). The histogram of such channel ensembles can give a discrete approximation of the PDF of the SNR at different bins and at the Rake combiner output SNR as described earlier. However, in order to observe the performance independent of total received energy variations, shadowing is eliminated while considering path loss model. Thus, with the knowledge of the discrete approximation of SNR distribution (PDF) and appropriate conditional error probability, the ABER of UWB system for Bi-orthogonal Keying (BOK) has been evaluated for all three Rake structures. An efficient coding/modulation method that can be easily applied to UWB system is M-ary BOK and its conditional BER can be given [9] as

$$
\begin{aligned}
& P_{e} E / \gamma=\frac{M}{2 M-1}\left(1-\frac{1}{\sqrt{2 \pi}}\right) \times \\
& \int_{-\infty}^{+\infty}\left(1-\frac{1}{2} \operatorname{erfc}\left(\left(x+\sqrt{\frac{2 M \gamma \log _{2} M}{M-1}}\right) \sqrt{\frac{1}{2}}\right)\right)^{M-1} e^{-\frac{x^{2}}{2}} d x
\end{aligned}
$$

The ABER versus average SNR per bit of $4 \mathrm{BOK}$ modulation for different Rake fingers and channel categories (CM1, CM2 and CM3) have been plotted in Figures 10,11 and 12. ABER of reduced complexity PRake and SRake structures are plotted and compared with ideal ARake structure. As anticipated, ARake has the best performance and taken as reference for comparison of SNR loss in PRake and SRake. Two and sixteen finger SRake \& PRake structures in different channel categories has been considered. The performance gain of SRake is found to be negligible in realistic CM1 channel, due to frequent presence of strong LOS component in the first delay bin. The performance gap of similar structures for even 16-fingers also remains nearly same. However, the performance loss of PRake compared to SRake increases appreciably in CM2 and CM3 channels. This is due to relatively pronounced degradation of PRake performance in CM2 and CM3. The difference in PRake and SRake curves increases in CM2 as fewer strong components are available in the beginning of temporal axis and the energy spread is over larger number of resolvable paths. For 16-finger PRake performance loss reduces enormously in both $\mathrm{CM} 2$ and $\mathrm{CM} 3$. Thus, ABER performance using the realistic channel model favours simple PRake 
structure in all CM1, CM2 \& CM3 channel categories with optimum number of Rake taps.

\section{CONCLUSIONS}

A semi-analytical evaluation of the realistic high data rate UWB error rate performance was done using Exponential-Lognormal indoor channel model. The PDF of output SNR distributions of SRake and PRake detectors has been investigated for CM1, CM2 and CM3 channel scenarios. The performance of reduced complexity Rake receivers in high data rate UWB indoor channels has been evaluated. In particular, we have used a PRake structure, which exploits only the first arriving multipath components and are usually less complex than conventional Rake receivers, since they do not require complete channel estimation. The performance of the PRake has also been compared with more complex SRake in UWB channels in terms of outage probability and ABER. Two fingers SRake outperforms the PRake for a large transmission bandwidth because performance gap between SRake and PRake widens in CM2 and CM3 channel conditions, as the first \& second multipath components may not be the strong components. However, the performance loss of PRAKE in these channels is shown to reduce appreciably by incorporating only 16 taps. Thus, on evaluating the important performance measures such as outage probability and ABER it is evident that the simpler PRake structure can be employed in rich diversity high data rate UWB channels with optimally selected fingers.

\section{REFERENCES}

[1] M. Z. Win and R. A. Scholtz, "On the robustness of ultra -wide bandwidth signals in dense multipath environments," IEEE Commun. Lett., vol. 2, pp. 51-53, Feb. 1998.

[2] M. Z. Win and R. A. Scholtz, "On the energy capture of ultra wide bandwidth signals in dense multipath environments," IEEE Commun. Lett., vol. 2, pp. 245-247, Sept. 1998.

[3] R.C. Qiu, H. Liu, and X. Shen, "Ultra-wideband for multipleaccess communications," IEEE Commun. Mag., vol.43, pp.8087, 2005.

[4] D. Cassioli, M. Z. Win, and A. Molisch, "The ultra-wide bandwidth indoor channel: From statistical model to simulations," IEEE J. Select. Areas Commun., vol. 20, no. 6, pp. 1247-1257, Aug. 2002.
[5] J. Foerster, "Channel modeling sub-committee report final," IEEE P802.15-02/490r1, Feb. 2003.

[6] A. F. Molisch, J. R. Foerster, and M. Pendergrass, "Channel models for ultra-wideband personal area networks," IEEE Wireless Commun. Mag., vol. 10, no. 6, pp. 14-21, Dec. 2003.

[7] S. S. Ghassemzadeh, L. J. Greenstein, T. Sveinsson, and V. Tarokh, "UWB delay profile models for residential and commercial indoor environments," IEEE Trans. Veh. Technol., vol. 54, no. 4, pp. 1235-1244, July 2005.

[8] Larry J. Greenstein, et al., "Comparison Study of UWB Indoor Channel Models ," IEEE transactions on wireless communications, vol. 6, no. 1, , pp. 128-135 January 2007.

[9] J. G. Proakis, Digital Communications, 4th ed. ed. New York: McGraw-Hill, 2001.

[10] J. Malhotra, A.K. Sharma and R.S. Kaler, "Investigation on Stochastic Tap Delay line Model of UWB Indoor Channel," in Proc. IEEE 5th International Conference on Electrical and Computer Engineering ICECE 2008, pp. 227-231, December 2008.

[11] J. Malhotra, A.K. Sharma and R.S. Kaler, "On the Performance of Rake Receivers for the UWB System in a Realistic Exponential-Lognormal Model," International Journal of Applied Engineering Research, vol.3, no.10, pp. 1287-1302, October 2008.

[12] H. Hashemi, "The indoor radio propagation channel," Proc. IEEE, vol. 81, no. 7, pp. 943-967, July 1993

[13] S. S. Ghassemzadeh, L. J. Greenstein, T. Sveinsson, and V. Tarokh., "UWB indoor path-loss model for residential and commercial buildings," in Proc. IEEE Semiannual Veh. Technol. Conf., vol. 5, pp. 3115 - 3119, Oct. 2003.

[14] A. F. Molisch, "Ultrawideband propagation channels - theory, measurement, and modeling," IEEE Trans. on Veh. Technol., vol. 54, pp. 1528-1545, Sept. 2005.

[15] Andrea Goldsmith, Wireless Communication, Cambridge University Press, 2005. 